\title{
Improvements to an optical scintillator imaging-based tissue dosimetry system
}

Irwin I. Tendler

Petr Bruza

Michael Jermyn

Antoine Fleury

Benjamin B. Williams

Lesley A. Jarvis

Brian W. Pogue

David J. Gladstone 


\title{
Improvements to an optical scintillator imaging-based tissue dosimetry system
}

\author{
Irwin I. Tendler, a, Petr Bruza, ${ }^{a}$ Michael Jermyn, ${ }^{\text {a,b }}$ Antoine Fleury, ${ }^{\text {a,c }}$ Benjamin B. Williams, ${ }^{\text {d,e }}$ Lesley A. Jarvis, ${ }^{\text {dee }}$ \\ Brian W. Pogue, ${ }^{\mathrm{a}, \mathrm{b}, \mathrm{e}}$ and David J. Gladstone $\mathrm{e}^{\mathrm{a}, \mathrm{d}, \mathrm{e}}$ \\ aDartmouth College, Thayer School of Engineering, Hanover, New Hampshire, United States \\ ${ }^{b}$ DoseOptics LLC, Lebanon, New Hampshire, United States \\ 'Université de Strasbourg, Télécom Physique Strasbourg, Illkirch-Graffenstaden, France \\ dDartmouth College, Geisel School of Medicine, Department of Medicine, Hanover, New Hampshire, United States \\ eNorris Cotton Cancer Center, Dartmouth-Hitchcock Medical Center, Lebanon, New Hampshire, United States
}

\begin{abstract}
Previous work has shown that capturing optical emission from plastic discs attached directly to the skin can be a viable means to accurately measure surface dose during total skin electron therapy. This method can provide accurate dosimetric information rapidly and remotely without the need for postprocessing. The objective of this study was to: (1) improve the robustness and usability of the scintillators and (2) enhance sensitivity of the optical imaging system to improve scintillator emission detection as related to tissue surface dose. Baseline measurements of scintillator optical output were obtained by attaching the plastic discs to a flat tissue phantom and simultaneously irradiating and imaging them. Impact on underlying surface dose was evaluated by placing the discs on-top of the active element of an ionization chamber. A protective coating and adhesive backing were added to allow easier logistical use, and they were also subjected to disinfection procedures, while verifying that these changes did not affect the linearity of response with dose. The camera was modified such that the peak of detector quantum efficiency better overlapped with the emission spectra of the scintillating discs. Patient imaging was carried out and surface dose measurements were captured by the updated camera and compared to those produced by optically stimulated luminescence detectors (OSLD). The updated camera was able to measure surface dose with $<3 \%$ difference compared to OSLD-Cherenkov emission from the patient was suppressed and scintillation detection was enhanced by $25 \times$ and $7 \times$, respectively. Improved scintillators increase underlying surface dose on average by $5.2 \pm 0.1 \%$ and light output decreased by $2.6 \pm 0.3 \%$. Disinfection had $<0.02 \%$ change on scintillator light output. The enhanced sensitivity of the imaging system to scintillator optical emission spectrum can now enable a reduction in physical dimensions of the dosimeters without loss in ability to detect light output. $\odot$ The Authors. Published by SPIE under a Creative Commons Attribution 4.0 Unported License. Distribution or reproduction of this work in whole or in part requires full attribution of the original publication, including its DOI. [DOI: 10 .1117/1.JBO.24.7.075001]
\end{abstract}

Keywords: scintillation; biomedical optics; camera; medical imaging; optical devices; radiation oncology.

Paper 190137R received Apr. 29, 2019; accepted for publication Jun. 24, 2019; published online Jul. 16, 2019.

\section{Introduction}

Previous research has demonstrated that imaging optical scintillation from plastic targets is a viable method of conducting surface dosimetry during radiotherapy. Surface dose information is obtained from light signals captured from multiple scintillator targets by an intensified and time-gated camera. This technique was designed to provide multipoint dosimetric information rapidly, remotely, and without the need for postexposure processing. ${ }^{1,2}$ This study focuses on quantifying improvements to both the scintillator dosimeter and camera components. Scintillators were redesigned to improve durability, ease-of-use, and chemical resistance of the plastic to cleaning procedures. The spectral sensitivity of the imaging system was optimized in order to maximize the signal-to-noise ratio (SNR) of scintillator light emission detection.

The intensified camera used in previous experiments ${ }^{1,2}$ was primarily designed for imaging Cherenkov light emissionthe photocathode was chosen to maximize detector quantum efficiency in the red wavelength ranges corresponding to

*Address all correspondence to Irwin I. Tendler, E-mail: Irwin.i.tendler.th@ dartmouth.edu
Cherenkov light production in animal and human tissues. ${ }^{3-9}$ Nevertheless, it has been shown that point surface dosimetry can still be accomplished owing to a sufficient overlap of the emission spectrum of the scintillator disks with the quantum efficiency spectrum of the camera photocathode. The camera has now been redesigned for detection of scintillator light emission, in turn, simultaneously enhancing scintillation and suppressing Cherenkov emission detection.

Scintillator dosimeters were originally designed to have a nonsoluble reflective coating on the rear face and side edges to allow for high light emissivity out of the front face and minimize angular dependency of the detected scintillation light. ${ }^{1,9-12}$ A water-based solvent compatible with the specific plastic composition of the scintillator was used to paint the scintillators. As such, the coated layer is mechanically fragile and chemically very susceptible to common medical-grade cleaning agents. To overcome this, earlier versions of the scintillator dosimeter required a single-use cellophane wrapper to enable attachment to the skin surface; thus, direct contact with the patient skin surface was avoided and the need for disinfection was eliminated. This approach, however, added an additional handling procedure during a routine clinical use; a redesign was issued in order to allow for disinfection and to eradicate the need for cellophane wrapping and handling. 
Considerations were made toward improving the mechanical and chemical hardness of the reflective coating: as an epoxybased paint could not be used due to typical dose-induced yellowing, and polyurethane-based solvents are not compatible with soft plastics, an oil-based protective layer was applied to the rear face and edge of the reflective paint-coated scintillator. This coating also helps protect the dosimeter, enabling more robust performance - potential signal loss from damage (e.g., paint chipping or surface scratches) is avoided. During redesign, the goal of direct attachment of the scintillator to the skin was established. To accomplish this, an adhesive backing, with minimal contact area, was utilized to attach the scintillator to the patient's skin. In this study, we present the impact of such modifications on both dosimetric output as well as clinical usability.

\section{Methods}

Much of the experimental methods and design have been described in two previous studies ${ }^{1,2}$ and so only the differentiating features are described here.

\subsection{Experimental Setup}

A batch of $n=10$ scintillator dosimeters were selected for this study. The scintillator discs $(15 \mathrm{~mm} \varnothing \times 1 \mathrm{~mm}$ thick $)$ were composed of EJ-212 plastic and custom-manufactured (Elijen Technologies, Sweetwater, Texas). The rear-faces of these discs were painted with EJ-510 reflective paint (Elijen Technologies). All 10 of the dosimeters were tested to obtain baseline measurements of light output, thickness, and influence on surface dose. A 500-193-30 AOS Digital Caliper (Mitutoyo, Aurora, Illinois) was used to measure dosimeter thickness. Varian $2100 \mathrm{CD}$ and Trilogy linacs (Varian Medical Systems, Palo Alto, California) were used for irradiation during phantom-based experiments and patient imaging sessions, respectively.

Five of the scintillators were kept unmodified as controls and the remaining five were modified with a protective coating and adhesive backing, according to the redesign aims - the modified scintillator group also underwent cleaning. These scintillators $(n=5)$ were coated with oil-based glossy clear-coat (Type 280702, Rust-Oleum, Vernon Hills, Illinois). Scintillators were cleaned using Super Sani-Cloth Germicidal Disposable Wipes (PDI, Orangeburg, New York). As per current clinical and manufacturer-recommend standards, the entire scintillator was thoroughly wiped and wet with the disposable wipes. The chemical cleaning agent was left on the dosimeter for $2 \mathrm{~min}$. Scintillator-sized $(0.18 \mathrm{~mm}$ thickness $\times 15 \mathrm{~mm} \varnothing)$ adhesive layers of 2477P double-coated thermoplastic elastomer silicone acrylate medical tape with premium liner (3M Maplewood, Minnesota) were placed on the rear faces of the modified scintillators to attach them to phantom and patient surfaces. A schematic of the redesigned scintillator is shown in the zoom-in panel of Fig. 1. The effect of protective coating and adhesive backing application on surface dose, dosimeter thickness, and light output were evaluated. A redesigned scintillator is shown side-by-side to a scintillator wrapped in cellophane in Fig. 1.

Following previously published methods, scintillators were placed directly on top of a calibrated PTW 23342 thin window ionization chamber (IC) (PTW, Freiburg, Germany) coupled to a Max 4000 electrometer (Standard Imaging, Middleton, Wisconsin) to evaluate the impact of the dosimeter thickness on surface dose. ${ }^{10}$ Samples were irradiated at $100 \mathrm{~cm}$ sourceto-surface distance $(\mathrm{SSD})$ using a $10 \mathrm{~cm} \times 10 \mathrm{~cm}$ 6-MeV electron field at 1000 monitor units (MU)/min. Dose rate was chosen

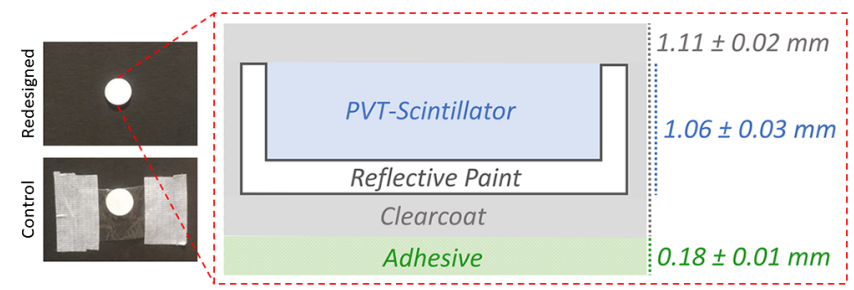

Fig. 1 Both "control" and "redesign" scintillator dosimeters mounted to a flat-faced phantom. "Control" scintillators are wrapped in cellophane and attached using medical tape. Zoomed-in view of the "redesigned" scintillator shows thicknesses of polyvinyl toluene (PVT) plastic, reflective paint, protective coating, and adhesive backing.

to minimize experiment time, scintillator output is independent of dose rate. ${ }^{10}$ For reference, the impact of a standard optically stimulated luminescence dosimeter (OSLD) nanoDOT (Landauer, Glenwood, Illinois) on surface dose was also tested. Percent change in surface dose caused by placement of a dosimeter on-top of the IC was calculated.

The methodology for conducting scintillator imaging-based surface dosimetry (including imaging setup specifications, image processing, dose conversion algorithm, etc.) has been previously described in detail. ${ }^{1,2,10}$ In general, a custom fitting algorithm is used to fit an ellipse-convolved Gaussian function to each scintillator within specified region-of-interest (ROI) per acquisition frame. The term, "light output," is defined as the sum of the maximum amplitudes of the fitted profiles across all frames. To collect light output measurements, scintillators were attached to a flat-faced acrylic resin phantom and irradiated with a 6-MeV high dose total skin electron beam ( $888 \mathrm{MU} / \mathrm{min})$. To mimic the patient imaging setup, the phantom was positioned using the geometry described below. Light output is converted to dose using an externally obtained calibration factor which accounts for Cherenkov light production in the disc, different calibration factors are necessary for "red"- and "blue"-sensitive cameras. $^{1,10}$

\subsection{Patient Imaging: Imaging System Modification}

Previous work demonstrated the feasibility of obtaining skin surface dose information for patients undergoing total skin electron therapy (TSET) by imaging light output of plastic scintillator targets using a linac-synchronized, time-gated, intensified camera system. ${ }^{1,2}$ In order to improve the sensitivity of this camera system to scintillator emission, the intensifier with redlight sensitive photocathode in the standard C-Dose camera (DoseOptics LLC, Lebanon New Hampshire) was replaced with one more sensitive to blue light. Otherwise, the chassis and design of the C-dose camera was not changed. For spectral sensitivity assessment, a calibrated tunable light source (Dynasil TLS-6, Newton, Massachusetts) was utilized. Measured spectral sensitivity was renormalized to quantum efficiency units using intensifier calibration records provided by the manufacturer (Photonis Technologies, Merignac, France). Comparative scintillator imaging was conducted on a patient being treated with TSET for a diagnosis of Mycosis fungoides. The patient was treated at a 3-m SSD and the camera was located $4 \mathrm{~m}$ away directly adjacent to the gantry head. Both red- and blue-sensitive camera systems were used to conduct surface dosimetry throughout the course of this patient's treatment following previously described methods. In order to facilitate a fair comparison to previous data, scintillators were attached to the patient 
using a cellophane wrapping without addition of a protective coating. TSET scintillators were attached to the upper arm, lower arm, chest, mid-section, thigh, shin, and foot. ${ }^{1}$

\section{Results and Discussion}

\subsection{Dosimeter Redesign}

\subsubsection{Thickness and surface dose measurements}

Prior to application of the protective coating, average scintillator thickness (including reflective paint) for both control and redesign groups was $1.06 \pm 0.03 \mathrm{~mm}$. Addition of the protective coating was found to add an additional $0.11 \pm 0.02 \mathrm{~mm}$ in thickness to the dosimeters. Compared to baseline IC surface dose measurements (shown as a green dot with corresponding standard deviation, STD, as vertical bar, Fig. 2), premodification control and redesign groups both showed an average $4.9 \pm 0.1 \%$ increase (blue and red horizontal dotted lines, Fig. 2). For reference, a standard OSLD was found to increase surface dose by an identical $4.9 \pm 0.1 \%$ (cyan star, Fig. 2). Following application of an adhesive backing, the postmodification redesigned scintillators increased the surface dose on average by $5.2 \pm 0.1 \%$ and $0.3 \pm 0.1 \%$, relative to IC baseline and OSLD, respectively. Thus, it was concluded that the impact on surface dose following addition of a protective coating and adhesive backing is comparable to that of an OSLD.

\subsubsection{Light output testing and dosimeter cleaning}

All scintillators were attached to a flat-faced phantom, left panel of Fig. 1. Control group scintillators were not removed in between steps of the modification and cleaning process; however, redesigned dosimeters were removed and reattached in

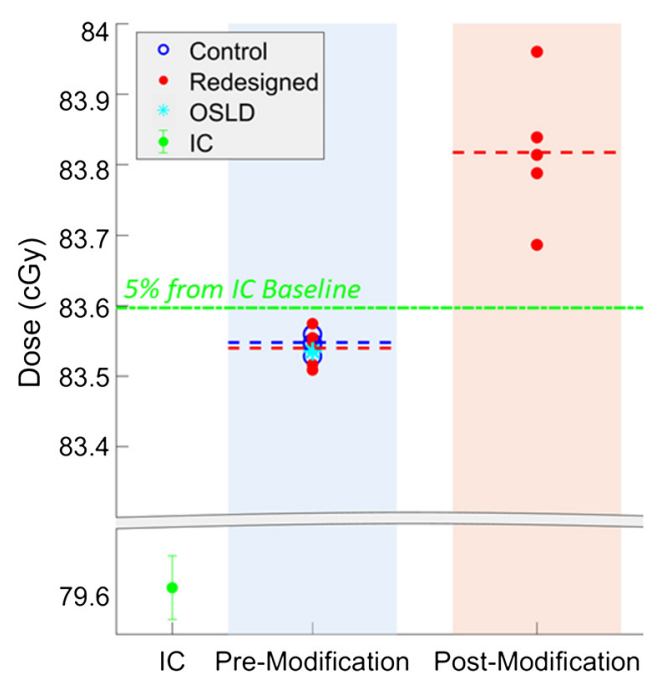

Fig. 2 Impact of scintillator $(n=10)$ thickness on surface dose. Reading obtained with bare IC, no dosimeters placed on-top shown as green dot, vertical green bar represents STD in IC measurements. $5 \%$ increase in surface dose from this baseline measurement shown as horizontal dotted green line. Pre- and postmodification refers to redesign process: application of clear coat and adhesive backing. "Redesigned" group of scintillators underwent the redesign process, while the "control" group did not. It should be noted that the $n=5$ "redesigned" scintillators in the pre- and postmodification sections are the same dosimeters. Cyan star shows impact of a nanoDot OSLD on surface dose. Horizontal dotted lines in each section represent mean of corresponding sample groups.

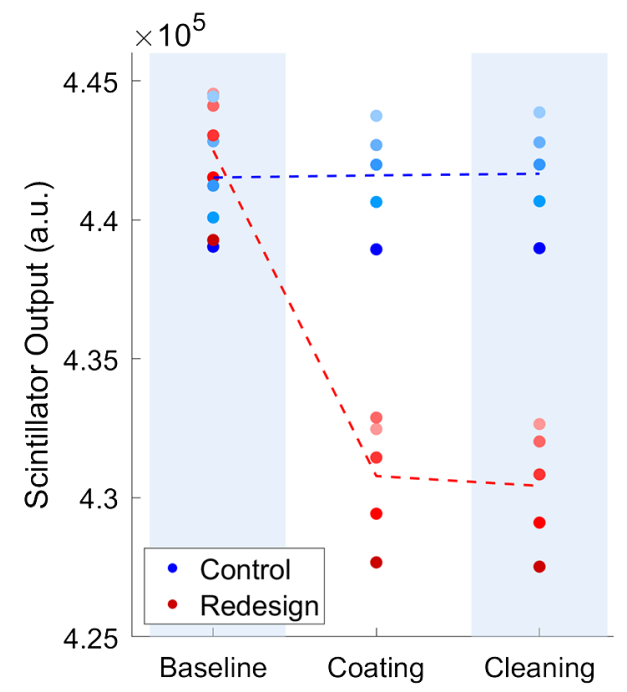

Fig. 3 Scintillator light output tracked over various stages of the redesigning process. Varying shades of red and blue represent individual scintillators. Mean of each sample group is represented as a colorcoded dotted line. "Coating" and "cleaning" represent light output measurement obtained following application of protective coating and cleaning with Sani-Cloth, respectively. Control group was not removed from the flat-faced phantom, and this group did not undergo coating or cleaning.

between stages. Modified scintillators were measured before and after application of the protective coating and cleaning procedures, these dosimeters were attached using an adhesive backing during all stages. For the control group, light output was found to change on average $<0.02 \%$ in between imaging sessions. An average $2.6 \pm 0.3 \%$ decrease in light output was measured following application of the protective clear coat when compared to baseline. A $<0.02 \%$ decrease in light output for modified scintillators was noted following the cleaning procedure, Fig. 3. As such, it was determined that light output from redesigned scintillators was not affected by cleaning with Sani-Cloth wipes.

\subsection{Imaging System Modification}

\subsubsection{Improved sensitivity to scintillation}

The spectra of light emitted from the scintillator dosimeter ranges from $\sim 400$ to $515 \mathrm{~nm}$, where the wavelength of maximal emission was found to be $422 \mathrm{~nm} .{ }^{10}$ A photocathode with overlap between maximum quantum detection efficiency and scintillator emission wavelength was selected. The quantum efficiency of the sensors was measured and is shown in Fig. 4-absolute response and coarse spectral profiles were measured by the photocathode manufacturer (Photonis, Merignac, France), while the fine relative spectra was measured by our research team. Increasing the sensitivity to scintillation emission can potentially allow for the use of smaller and thinner scintillating volumes during scintillation dosimetry. This would be especially important during treatment scenarios where minimizing field perturbation is more critical, such as when the field size is smaller and dose gradient is larger. Cherenkov emission produced during treatment is useful for monitoring and verifying radiation field geometry. ${ }^{13}$ Despite that the "blue"-sensitive camera suppresses Cherenkov light detection, one can still visualize Cherenkov emission signal by window and leveling in realtime using the camera acquisition CDose software. 


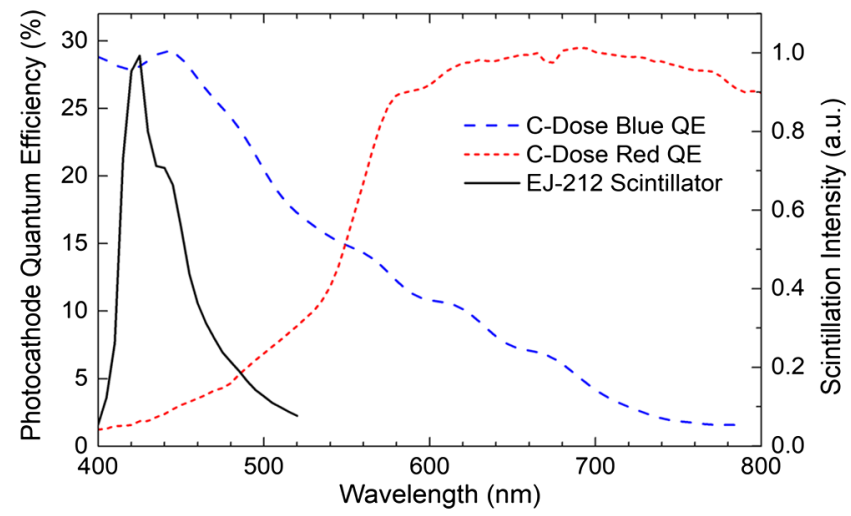

Fig. 4 Emission spectra of the scintillator dosimeter ${ }^{15}$ (black solid line, right axis) overlaid with quantum detection efficiency spectra of the red- and blue-sensitive photocathodes (red and blue dashed lines, data correspond to left axis).

\subsubsection{Patient imaging}

Scintillator dosimetry was conducted over the course of six TSET treatment positions (anterior-posterior, posterior-anterior, left and right-anterior-oblique, left and right-posterior oblique)

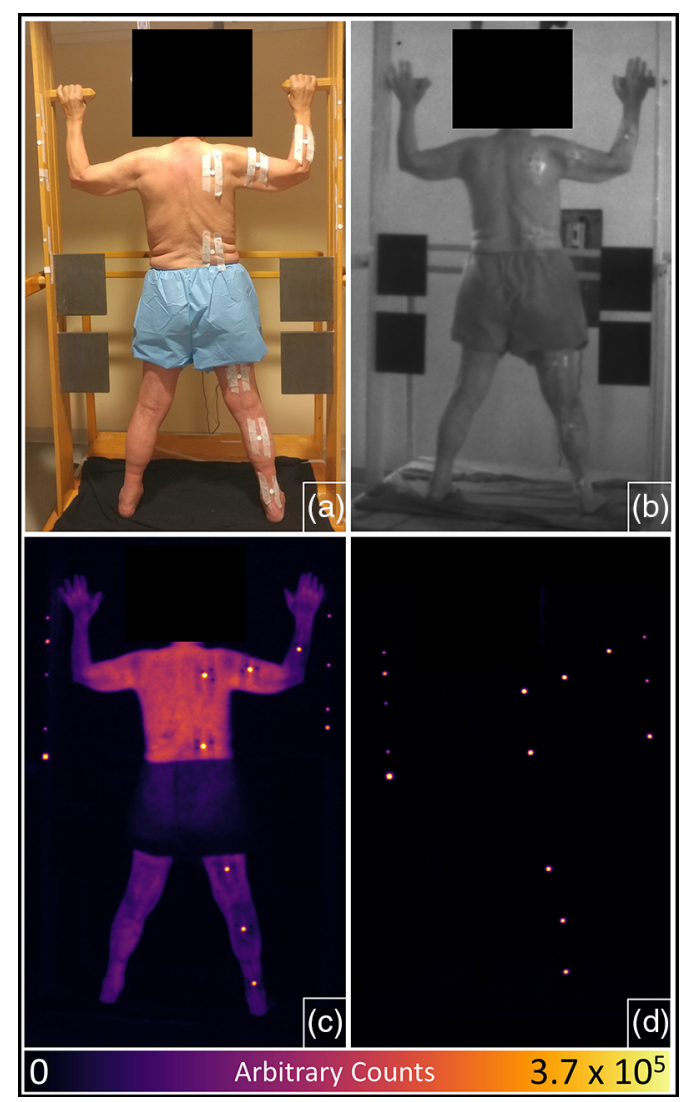

Fig. 5 Sample images of a patient undergoing TSET in the posterioranterior position. (a) Color photograph, (b) background image, (c) cumulative image captured by "red"-camera, and (d) cumulative image captured simultaneously during the same imaging session as shown in (c) by "blue" camera. Intensity values of Cherenkov and scintillation intensity maps shown in (c) and (d) are identically scaled. The SNR of the lower back scintillator (scintillation signal) and $50 \times$ 50-pixel square $\mathrm{ROI}$ in the center of back (Cherenkov light) increased and decrease by $25 \times$ and $7 \times$ when comparing (c) to (d), respectively. for both "red"- and "blue"-sensitive cameras. Sample images for the anterior-posterior position are provided in Fig. 5. Previous reports provide details regarding the intensity distribution across the scintillators, fitting profiles, and associated errors. ${ }^{1,10}$ Scintillators were attached to seven dosimetry sites across the body, in two cases during imaging with the "blue"-sensitive camera, scintillators were removed from the foot due to patient compliance issues. Thus, data from dosimetry sites (80 total) were considered for analysis. For reference, each scintillator was paired with an OSLD. Comparing dose measured by OSLD to scintillator, per dosimetry site, it was found that a linear relationship existed for both "red" and "blue" imaging systems, $R^{2}=$ 0.96 and 0.95 , respectively. These data points are plotted for this patient (PT6), as well as others evaluated over the course of the last 1.5 years in a human pilot study. ${ }^{1}$ In total, data for $n=6$ patients and 242 dosimetry sites are provided in Fig. 6. Data showed that irrespective of whether the red- or blue-sensitive camera was used, accurate surface dosimetry was achieved. The percent difference in dose measured by OSLD and scintillator was found to be $<5 \%$ and $<3 \%$ for $241 / 242$ and $221 / 242$ sites, see Fig. 6.

\section{Conclusion}

Radioluminescence imaging has been developing as a noncontact imaging tool to allow for quantitative dosimetry for several years. Advances in imaging Cherenkov from tissue have been
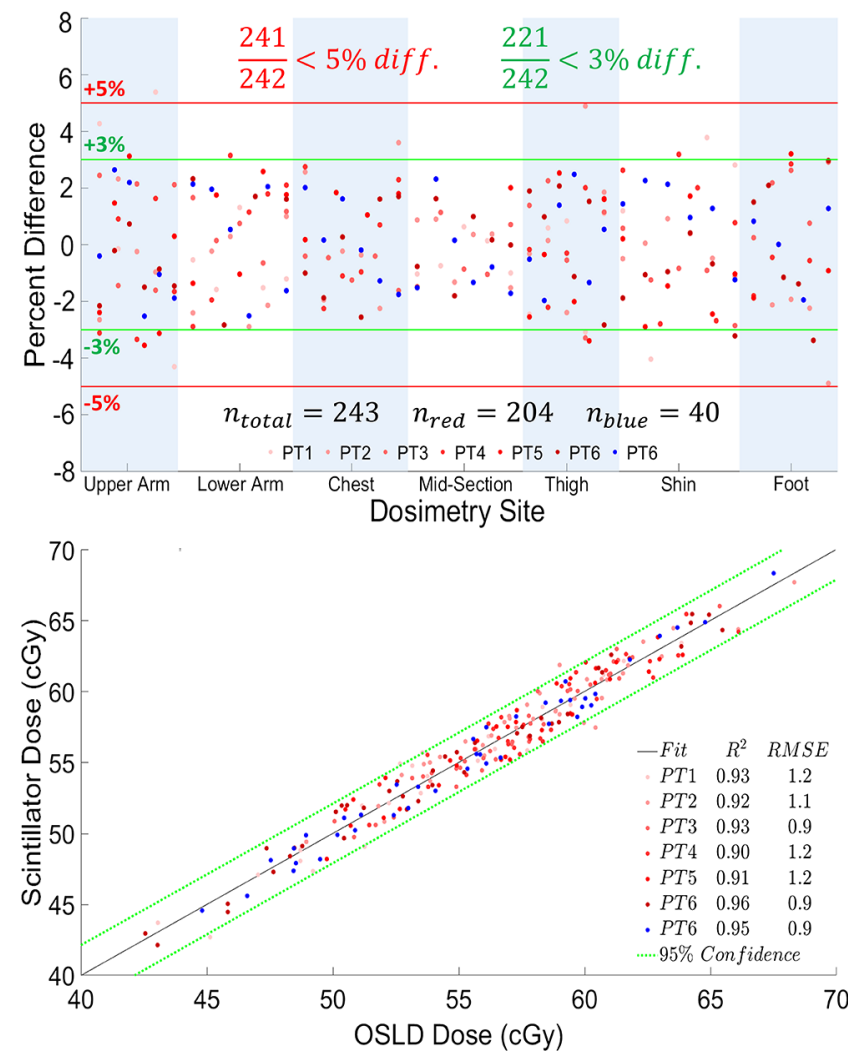

Fig. 6 (a) Percent difference $( \pm 3 \%=$ green and $\pm 5 \%=$ red) between surface dose measured by scintillator and OSLD, per dosimetry site. (b) Relationship between surface dose measured by scintillator versus OSLD. Linear trendline and $95 \%$ confidence interval are displayed in black and green, respectively. Data points obtained by red- and blue-sensitive cameras are shown with different shades of red or blue color, respectively. $R^{2}$ and root-mean-square error for each patient data set are also shown. 
important to visualize radiation delivery, but still the emission from tissue shows light that is attenuated by the tissue optical properties. In comparison, imaging scintillation provides a direct dose reporter if the optical signal can be detected in a manner that is independent of the light interaction with tissue. ${ }^{14}$ This approach to scintillator-based surface dosimetry has been shown to allow accurate dose estimation, and the modifications introduced here will improve both the detection sensitivity and actual dosimeter function as a biomedical tool. The application of a protective coating and adhesive backing helps streamline the dosimeter application process, allows for cleaning of the dosimeter, and reduces potential damage that is possible from normal everyday use. The improved blue-sensitive camera used in this scintillation dosimetry system has been altered such that, relative to the previous version, the SNR of Cherenkov emission detected from tissue is suppressed, and the scintillation signal is enhanced, by $25 \times$ and $7 \times$, respectively. This results because of the redshift of Cherenkov light emitted from tissue, due to blood absorption of the blue/green wavelengths. With these improved detection specifications, it is possible that the physical dimensions of the scintillator could now be reduced, or the camera position could be increased in distance for the same SNR. These changes to increase detection of light output fit the clinical needs of treatments requiring minimal field perturbation and minimal workflow interruption.

\section{Disclosures}

All studies have been approved by the Dartmouth-Hitchcock Medical Center Internal Review Board and informed consent was obtained from all patients. M. Jermyn is an employee and B. Pogue is a president of DoseOptics LLC. P. Bruza is a principal investigator in SBIR subaward B02463 (prime award NCI R44CA199681, DoseOptics LLC). The other authors have no relevant financial interests in this article and no potential conflicts of interest to disclose.

\section{Acknowledgments}

This work was sponsored by NIH Grant No. R01 EB023909. Supply of equipment and software for the study was provided by DoseOptics LLC under NIH Grant No. R44 CA199836. Irradiation Shared Resources from the Norris Cotton Cancer Center Grant No. P30 CA023106.

\section{References}

1. I. Tendler et al., "Rapid multi-site remote surface dosimetry for total skin electron therapy: scintillator target imaging," Int. J. Radiat. Oncol. 103(3), 767-774 (2019).

2. P. Bruza et al., "Time-gated scintillator imaging for real-time optical surface dosimetry in total skin electron therapy," Phys. Med. Biol. 63(9), 095009 (2018).

3. J. M. Andreozzi et al., "Improving treatment geometries in total skin electron therapy: experimental investigation of linac angles and floor scatter dose contributions using Cherenkov imaging," Med. Phys. 45(6), 2639-2646 (2018).

4. Y. Xie et al., "Cherenkov imaging for total skin electron therapy (TSET)," Proc. SPIE 10478, 1047816 (2018).

5. R. Hachadorian et al., "Correcting Cherenkov light attenuation in tissue using spatial frequency domain imaging for quantitative surface dosimetry during whole breast radiation therapy," J. Biomed. Opt. 24, 071609 (2018).

6. T. Miao et al., "Cherenkov imaging for linac beam shape analysis as a remote electronic quality assessment verification tool," Med. Phys. 46(2), 811-821 (2018).
7. C. Snyder et al., "Algorithm development for intrafraction radiotherapy beam edge verification from Cherenkov imaging," J. Med. Imaging 5, 015001 (2018).

8. L. A. Jarvis et al., "Cherenkov video imaging allows for the first visualization of radiation therapy in real time," Int. J. Radiat. Oncol. 89, 615-622 (2014).

9. R. Zhang et al., "Real-time in vivo Cherenkoscopy imaging during external beam radiation therapy," J. Biomed. Opt. 18, 110504 (2013).

10. I. I. Tendler et al., "Characterization of a non-contact imaging scintillator-based dosimetry system for total skin electron therapy," Phys. Med. Biol. 64(12), 125025 (2019).

11. I. I. Tendler et al., "Technical note: quality assurance and relative dosimetry testing of a60Co total body irradiator using optical imaging," Med. Phys. (2019).

12. D. A. Alexander et al., "Assessment of imaging Cherenkov and scintillation signals in head and neck radiotherapy," Phys. Med. Biol. (2019).

13. R. Zhang et al., "Cherenkoscopy based patient positioning validation and movement tracking during post-lumpectomy whole breast radiation therapy," Phys. Med. Biol. 60, L1-L14 (2015).

14. B. W. Pogue and B. C. Wilson, "Optical and X-ray technology synergies enabling diagnostic and therapeutic applications in medicine," J. Biomed. Opt. 23, 121610 (2018).

15. Eljen Technology, EJ-200, EJ-204, EJ-208, EJ-212, 2016. https:// eljentechnology.com/products/plastic-scintillators/ej-200-ej-204-ej-208ej-212

Irwin I. Tendler is a PhD candidate at the Thayer School of Engineering at Dartmouth College. He is interested in the development of medical physics-related biomedical imaging systems and has experience in both clinical and bench-top research settings. His work is centered on the use of imaging scintillation light for dosimetry and quality assurance applications in radiation oncology. He is a two-time winner of the American Association Physicists in Medicine Young Investigator Symposium competition.

Petr Bruza is an assistant professor in engineering sciences at Dartmouth College. His research interests include optics in medicine, biomedical imaging to guide cancer therapy, dose imaging in radiation therapy, and Cherenkov light imaging. His work entitled "3D Cherenkov Sheet Molecular Imaging provides 100-micron whole body spatial resolution" was awarded the "Best in Physics" designation for imaging physics.

Michael Jermyn is an adjunct assistant professor at the Thayer School of Engineering, Dartmouth College. He is an expert in computational solutions for biomedical applications. He has experience in machine learning, computer vision, software development and developing tools for surgical guidance, cancer imaging, therapies, and treatment monitoring.

Antoine Fleury is a recent graduate from the University of Strasbourg achieving both undergraduate and graduate degrees in photonics science. He has conducted research in analyzing spectral output of scintillation and Cherenkov light emission for optics in medicine applications.

Benjamin B. Williams is an associate professor of medicine and radiology at the Geisel School of Medicine and adjunct associate professor of engineering at the Thayer School of Engineering at Dartmouth College. He is also an author of over 60 publications and a practicing medical physicist at the Norris Cotton Cancer Center at DartmouthHitchcock Medical Center.

Lesley A. Jarvis is an associate professor of medicine at the Geisel School of Medicine at Dartmouth, and a practicing radiation oncologist at the Norris Cotton Cancer Center at Dartmouth-Hitchcock Medical Center. She is author of over 70 publications and has overseen the translation and implementation of Cherenkov imaging for real-time field verification and scintillation dosimetry in the clinical workflow at DHMC for cancer therapy monitoring.

Brian W. Pogue is the MacLean Professor of Engineering Sciences at Dartmouth College, director of MS and PhD programs, as well as adjunct professor of surgery at the Geisel School of Medicine. He has published over 330 peer-reviewed papers and $>400$ conference 
papers in the areas of monitoring cancer therapy with optical signals, surgery, medicine, medical oncology, and radiotherapy. Finally, he is the cofounder and president of the company DoseOptics LLC.

David J. Gladstone is a professor of medicine at the Geisel School of Medicine, an adjunct professor of engineering at the Thayer School of
Engineering at Dartmouth, and the chief of clinical physics at the Norris Cotton Cancer Center. His professional interests include ultraconformal radiation therapy to spare normal tissues from damage secondary to treatment, image guided radiation therapy, biological gating of therapeutic $x$-ray beams, image guided brachytherapy, Cherenkov emission during radiotherapy, and EPR dosimetry. 